\title{
Re: The relationship between patient and tumor characteristics, patterns of breast cancer care, and 5-year survival among elderly women with incident breast cancer. Breast Cancer Res Treat. Sep 2018;171(2):477-488
}

\author{
Elvin S. Cheng ${ }^{1,2} \cdot$ Xue Qin $\mathrm{Yu}^{1,2}$
}

Received: 8 December 2018 / Accepted: 12 December 2018 / Published online: 4 January 2019

c) Springer Science+Business Media, LLC, part of Springer Nature 2019

\section{Dear Editor,}

We read with interest the recent article by Kong et al. published in your journal in which the authors assessed the relationship between patient and tumor characteristics, patterns of care, and 5-year survival in a population-based cohort of women diagnosed with breast cancer. An additional aim was to identify possible reasons for disparities in survival. They used a Classification and Regression Tree (CART) model which provided insights into the patterns of factors that classify patients into groups with similar survival. They also showed that compared with the high socioeconomic status (SES) group, those in the lower SES group were more likely to have patterns associated with the lowest survival than with the highest survival.

However, we would like to point out a few errors in the article. Firstly, some results were incorrectly presented in the Appendix Table 3: while the results in the "\% Alive" column in Table 2 were correctly shown, the results in the "\% 5-year survival" column should have been 100 minus the estimates presented in Table 3. Secondly, the column "Unknown" under Tumor size (\%) in Table 3 presented the value of zero (0) for all patterns of care. This appears to be an error as the proportion of the unknown should be the remaining percentage after subtracting the total of the 3 groups of tumor size from 100. Lastly, the two-year survival as mentioned in the second paragraph of the discussion (p. 482) should be fiveyear survival as analyzed in this study.

Additional minor changes may also improve the presentation of the results. The terms "Medium income" and "High income" were used in Tables 1 and 3 while the terms "Medium SES" and "High SES" were used in the main Table (Table 2) of the article. As the indicator of SES used is a composite measure and is not just based on personal income, a consistent use of "Poor," Near poor," "Medium SES," and "High SES" would be preferable. Also, some of the results under the column "Median positive nodes" in Table 3 were quite unusual (especially for pattern 14 where 100 was reported) and it would be good to check its validity. Lastly, in our opinion, it would be worthwhile to compare their results/interpretation of CART with that from a standard analysis using Cox proportional hazards regression.

Funding This letter has no specific funding for it. Dr Elvin Cheng is a PhD candidate of the University of Sydney, and Dr Xue Qin Yu is employed by the Cancer Council NSW, Australia.

\section{Compliance with ethical standards}

Conflict of interest We solemnly declare that we have no conflict of interest.

This Letter to the Editor refers to the article available online at https://doi.org/10.1007/s10549-018-4837-4.

Elvin S. Cheng

elvin.cheng@nswcc.org.au

1 Cancer Research Division, Cancer Council NSW, Sydney, Australia

2 The University of Sydney School of Public Health, Sydney, Australia 